\title{
Beam transformations by active selfoc microlenses
}

\section{Transformaciones de haz mediante microlentes selfoc activas}

\author{
Ana I. Gomez-Varela(1), María Teresa Flores-Arias(1,S), Carmen Bao-Varela(1), \\ Xermán de la Fuente ${ }^{(2)}$, Carlos Gomez-Reino(1,S, $\left.{ }^{*}\right)$ \\ 1. Grupo de "Microóptica y Óptica GRIN", Unidad Asociada al Instituto de Ciencias de Materiales de Aragón, \\ ICMA/CSIC, Zaragoza, Facultade de Óptica e Optometría e Facultade de Física, Campus Vida s/n, Universidade de \\ Santiago, E15782 Santiago de Compostela, Spain. \\ 2. Instituto de Ciencia de Materiales de Aragón (Universidad de Zaragoza-CSIC), María de Luna 3, E50018 Zaragoza, \\ Spain. \\ (*) Email: carlos.gomez-reino@usc.es \\ S: miembro de SEDOPTICA / SEDOPTICA member \\ Recibido / Received: 30/09/2011. Revisado / Revised: 15/02/2012. Aceptado / Accepted: 20/02/2012.
}

\begin{abstract}
:
In this paper light propagation in an active selfoc microlens with complex refractive index and gradient parameter are examined. The ray-transfer matrix of an active material regarded as a diffraction-free first order optical system is presented to describe the beam transformations in active selfoc microlenses. Results concerning Gaussian beam transformations and on-axis irradiance through the active selfoc microlens are discussed and commented.
\end{abstract}

Key words: Active Material, Selfoc Microlens, GRIN Optics, Beam Transformation.

\section{RESUMEN:}

En este trabajo se estudia la propagación de la luz a través de una microlente selfoc activa con un índice de refracción y parámetro de gradiente complejos. La matriz de transferencia de rayos, vista como un sistema óptico de primer orden libre de efectos de difracción, es introducida para describir las transformaciones que puede sufrir un haz en microlentes selfoc activas. Por último se presentan y comentan los resultados correspondientes a las transformaciones de haces Gaussianos y a la irradiancia en eje a través de la microlente selfoc activa.

Palabras clave: Material Activo, Microlente Selfoc, Óptica GRIN, Transformación de Haz.

\section{REFERENCIAS Y ENLACES / REFERENCES AND LINKS}

[1]. C. Gomez-Reino, M. V. Perez, C. Bao, GRIN Optics: Fundamentals and Applications, Springer Verlag, Berlin (2002).

[2]. H. Kogelnik, "On the propagation of Gaussian beams of light through lenslike media including those with a loss or gain variation", Appl. Opt. 4, 1562-1569 (1965).

[3]. L. Casperson, A. Yariv, "The Gaussian mode in optical resonators with a radial gain profile", Appl. Phys. Lett. 12, 355-357 (1968).

[4]. S. Velumani, SA. K. Narayandass, D. Mangalara and C. P. Girija Vallaban, "Laser damage studies on hot wall deposited CdSe thin films", J. Mater Sci. Lett. 16, 1974-1976 (1997).

[5]. S. Velumani, SA. K. Narayandass, D. Mangalara, C. P. Girija Vallaban,"Linear pulse propagation in an absorbing medium: Effect of film thickness", Phys. Rev. B 49, 10742-10744 (1994).

[6]. R. K. Luneburg, Mathematical Theory of Optics, University of California Press, Berkeley (1964)

[7]. S. Velumani, SA. K. Narayandass, D. Mangalara, C. P. Girija Vallaban, "Beam characterization through active media", Opt. Commun. 85, 10742-10744 (1991). 


\section{Introduction}

Selfoc microlens is a sort of lossless gradientindex rod material in which the refractive index on transverse planes is described by a parabolic distribution. The index profile has a maximum at the center of the microlens (on-axis) and decreases quadratically towards the outer edge. Selfoc microlenses are ideal for many signal transmission and focusing applications as laser diode-to-fiber, fiber-to-detector and fiber-tofiber coupling, focusing and collimating [1]. In an active GRIN material a light beam experiences gain or loss. Effects of gain or loss in GRIN materials can be phenomenologically taken into account by using a complex refractive index. A GRIN rod that has both quadratic gain or loss and refractive index profiles in transverse planes away from the optical axis is known as an active selfoc microlens. Early discussions of propagation through quadratic materials with gain or loss were given in 1965 by Kogelnik [2] and a few years later by Casperson and Yariv [3]. The knowledge of the beam transformations for Gaussian beams propagating through active materials having loss or gain is necessary, for example, in material processing and laser damage studies [4] and thin films [5]. Among these beam transformations we have beam shaping, process of redistributing, in a controlled manner, the irradiance and phase of a beam optical radiation. A typical example of beam shaping is the conversion of a Gaussian laser beam into a beam with uniform amplitude and constant phase, that is, flat-top beam. To our knowledge, no beam shaping by active selfoc microlens has been reported. The purpose of the paper is to examine the implications of using a complex refractive index on light propagation in active selfoc microlenses in order to describe how Gaussian beams may be transformed by these microlenses and, in particular, how beam shaping and focusing take place. In this paper expressions for the complex refractive index and gradient parameters are examined to discuss what gain or loss means in terms of both. The correspondence between the linear integral transformation and the ray-transfer matrix of an active material regarded as a diffraction-free first order optical system is presented to describe the beam transformations in an active selfoc microlens. Results concerning on axis irradiance distribution and on beam shaping and focusing positions in active selfoc microlens are commented. At the end conclusions are given.

\section{Complex refractive index}

We consider an active selfoc microlens, with rotational symmetry around the $z$ axis, of thickness $d$ and semiaperture a limited by plane parallel faces whose refractive index can be written as a parabolic transverse gain or loss profile:

$$
n(r)=n_{0}\left(1-\frac{g_{0}^{2}}{2} r^{2}\right),
$$

for $r=\left(x^{2}+y^{2}\right)^{2} \leq a$ and $0 \leq z \leq d$, where $n_{0}$ and $g_{0}$ are the on-axis complex refractive index and the gradient parameter, respectively, given by:

$$
\begin{aligned}
& n_{0}=n_{0 R}+i n_{0 I}, \\
& g_{0}=g_{0 R}+i g_{0 I},
\end{aligned}
$$

with $n_{0 R}, n_{0 I}$ and $g_{0 R}, g_{0 I}$ being real constants.

From Eqs. (2) and (3) the real and imaginary part of the refractive index can be expressed by:

$$
\begin{aligned}
n_{R}=n_{0 R} & -\left\{\frac{n_{0 R}}{2}\left[g_{0 R}^{2}-g_{0 I}^{2}\right]\right. \\
& \left.-n_{0 I} g_{0 R} g_{0 I}\right\} r^{2} \\
n_{I}=n_{0 I} & -\left\{\frac{n_{0 I}}{2}\left[g_{0 R}^{2}-g_{0 I}^{2}\right]\right. \\
& \left.+n_{0 R} g_{0 R} g_{0 I}\right\} r^{2} .
\end{aligned}
$$

The real part of the refractive index $n_{R}$ determines the guidance behavior of the active material and the gain or loss in active selfoc lens is determined by the sign of the imaginary part of the refractive index $n_{I}$. The material has loss if $n_{I}>0$ and it experiences gain if $n_{I}<0$. The real part $g_{0 R}$ of gradient parameter determines the transverse parabolic profile and the imaginary part $g_{0 I}$ is related to the effect of gain or loss on this parabolic profile.

Figure 1 shows the profiles of real and imaginary parts of the refractive index for two cases, on-axis gain or loss, where the values considered correspond to commercial parameters used for the obtention of GRIN microlenses, being $n_{0 I}=-10^{-5}, n_{I}(a)=-10^{-4}$ (solid line) and $n_{0 I}=10^{-5}, n_{I}(a)=-10^{-4}$ 
(dashed line). Figure 1(a) represents the real part of the complex refractive index for the two cases considered, being the profiles very similar. Figure 1(b) shows the plotting of the imaginary parts of the index for on-axis gain and loss cases. In both of them a parabolic behaviour is shown, being the values obtained for the center higher than at the edge. In on-axis gain case, the imaginary part of the refractive index is negative and a concentration of energy around the axis occurs. However, in on-axis loss case, the sign of the imaginary part of the refractive index changes from positive to negative values in such a way that energy spreads to the edges of the active microlens. In all cases a semiaperture value of the active microlens of $0.5 \mathrm{~mm}$ has been considered.
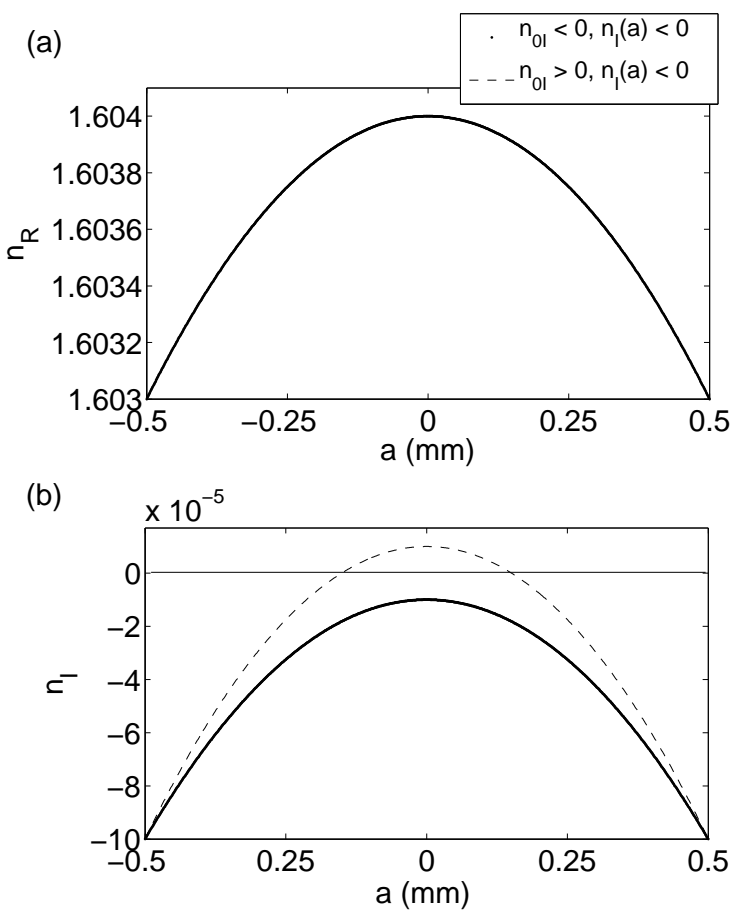

Fig. 1: Profiles of (a) real and (b) imaginary part of the complex refractive index for an active selfoc microlens with $a=0.5 \mathrm{~mm}, n_{0 R}=1.604$ and $n_{R}(a)=1.603$, exhibiting onaxis gain (solid line) and on-axis loss (dashed line). Parameter values: on-axis gain case $n_{0 I}=-10^{-5}$, $n_{I}(a)=-10^{-4}, g_{0 R}=0.070694 \mathrm{~mm}^{-1}$ and $g_{0 I}=0.003175$ $\mathrm{mm}^{-1}$; on axis-loss $n_{0 I}=10^{-5}, \quad n_{I}(a)=-10^{-4}, \quad g_{0 R}=$ $0.070729 \mathrm{~mm}^{-1}$ and $g_{0 I}=0.003878 \mathrm{~mm}^{-1}$

\section{Light propagation in an active selfoc microlens}

The active selfoc microlens operating in the paraxial domain with complex refractive index given by Eq. (1) is regarded as an active first- order system [1]. The complex matrix equation for ray propagation, in this case, is expressed as:

$$
\begin{aligned}
& \left(\begin{array}{c}
q\left(z_{1}\right) \\
n_{0} \dot{q}\left(z_{1}\right)
\end{array}\right)= \\
& \quad=\left(\begin{array}{cc}
H_{f}\left(z_{1}\right) & \frac{H_{a}\left(z_{1}\right)}{n_{0}} \\
n_{0} \dot{H}_{f}\left(z_{1}\right) & \dot{H}_{a}\left(z_{1}\right)
\end{array}\right)\left(\begin{array}{c}
q(0) \\
n_{0} \dot{q}(0)
\end{array}\right) .
\end{aligned}
$$

Equation (6) describes the position, $q\left(z_{1}\right)$ and the slope, $\dot{q}\left(z_{1}\right)$, of any complex ray at $z_{1}$ in the material in terms of the position and slope of the complex ray at the input face of the microlens. The position and the slope at $z_{1}$ can be expressed as a linear combination of the axial and field complex rays. $H_{a}$ and $\dot{H}_{a}$ represent the position and the slope of the complex axial ray at $z_{1}$ and $H_{f}$ and $\dot{H}_{f}$ are the position and the slope of the complex field ray at $z_{1}$ [6]. We suppose now that an active selfoc microlens is illuminated by a monochromatic Gaussian beam of wavelength $\lambda$ and beam waist of diameter $2 \omega_{0}$ located at distance $d_{1}$ from the input face. Gaussian beam propagation through this microlens can be described by a linear integral transformation given by [1]:

$$
\begin{aligned}
& \Psi\left(x_{1}, y_{1} ; z_{1}\right)= \\
& =\int_{R^{2}} K\left(x_{0}, y_{0}, x_{1}, y_{1} ; z_{1}\right) \Psi\left(x_{0}, y_{0} ; 0\right) d x_{0} d y_{0} .
\end{aligned}
$$

where $\Psi\left(x_{0}, y_{0} ; 0\right)$ is the complex amplitude distribution of the Gaussian beam at the input plane and $K$ is the Point Spread Function of the active material.

$$
\begin{aligned}
& \Psi\left(x_{1}, y_{1} ; z_{1}\right)= \\
& \quad=\frac{\omega_{0} e^{-k n_{0 I} z_{1}} e^{i k n_{0 R} z_{1}}}{\omega(0) \alpha\left(z_{1}\right)} e^{i \frac{\pi U\left(z_{1}\right)}{\lambda}\left(x_{1}^{2}+y_{1}^{2}\right),}
\end{aligned}
$$

where $U\left(z_{1}\right)$ is the complex curvature at $z_{1}$, expressed by the position and the slope of the complex ray as:

$$
U\left(z_{1}\right)=n_{0} \dot{q}\left(z_{1}\right) q^{-1}\left(z_{1}\right)
$$

and

$$
\alpha\left(z_{1}\right)=H_{f}\left(z_{1}\right)+\frac{U(0)}{n_{0}} H_{a}\left(z_{1}\right),
$$

$U(0)$ and $\omega(0)$ being the complex curvature and the beam half-width at the input face.

Equation (9) can be used for evaluating beam parameters at $z_{1}$ by the complex ray since the 
complex curvature in terms of the radius of curvature and beam half-width says:

$$
\begin{aligned}
U\left(z_{1}\right)= & U_{R}\left(z_{1}\right)+i U_{I}\left(z_{1}\right)= \\
= & \frac{1}{R\left(z_{1}\right)}+i \frac{\lambda}{\pi \omega^{2}\left(z_{1}\right)} .
\end{aligned}
$$

Comparing Eqs. (9) and (11), the beam parameters at $z_{1}$ can be obtained:

$$
\begin{aligned}
R\left(z_{1}\right) & =\frac{\left|q\left(z_{1}\right)\right|^{2}}{\operatorname{Re}\left[n_{0} \dot{q}\left(z_{1}\right) q^{*}\left(z_{1}\right)\right]}, \\
\omega^{2}\left(z_{1}\right) & =\frac{\lambda\left|q\left(z_{1}\right)\right|^{2}}{\pi \operatorname{Im}\left[n_{0} \dot{q}\left(z_{1}\right) q^{*}\left(z_{1}\right)\right]} .
\end{aligned}
$$

Note that the Gaussian beam is not $z$ invariant when considering propagation through the active selfoc microlens since the z-invariant condition does not hold for the complex rays:

$$
q^{*}\left(z_{1}\right) \dot{q}\left(z_{1}\right)-q\left(z_{1}\right) \dot{q}^{*}\left(z_{1}\right) \neq \text { constant, }
$$

for $d>z_{1}>0$.

This fact establishes an important difference from the lossless or passive GRIN materials, in which the beam half-width is expressed as the modulus of the complex ray and the wavefront is perpendicular to the beam profile [7].

The irradiance at $z_{1}$ can be easily obtained from Eq. (8):

$$
I\left(x_{1}, y_{1} ; z_{1}\right)=I_{0} \frac{e^{-2 k n_{0 I} z_{1}}}{\left|\alpha\left(z_{1}\right)\right|^{2}} e^{\frac{-2\left(x_{1}^{2}+y_{1}^{2}\right)}{\omega^{2}\left(z_{1}\right)}},
$$

where $I_{0}=\omega_{0}^{2} / \omega^{2}(0)$.

Therefore, a Gaussian irradiance profile is obtained at $z_{1}$ with on-axis loss or gain determined by the sign of the imaginary part $n_{0 I}$ of the refractive index along $z_{1}$ axis. Depending on the sign of the imaginary part in Eq. (13), the beam half-width square, $\omega^{2}$, can be either positive or negative. If $\omega^{2}$ is positive, a Gaussian beam is achieved whose irradiance, according to Eq. (15), is a maximum at axis and decreases with radial distance. On the contrary, if $\omega^{2}$ is negative, a Gaussian beam is obtained whose irradiance is a minimum along the $z_{1}$-axis and increases with radial distance. Therefore, light concentration or light spread around the $z_{1}$-axis occurs as $\omega^{2}$ is positive or negative, respectively.

\section{Beam transformations}

The beam half-width evolution of a He-Ne laser operating at $632.8 \mathrm{~nm}$ is shown in Fig. 2. Figure 2(a) corresponds to on-axis gain case and Fig. 2(b) corresponds to on-axis loss case. Both present very slight changes in their behaviour except for the region around $\omega^{2}$ changes its sign, in other words, where the Gaussian beam becomes a uniform beam obtaining beam shaping (flat-top beam).

Figure 3 shows the behaviour of the radius of curvature of the Gaussian beam as it propagates through the active microlens for on axis-gain (a)

(a)

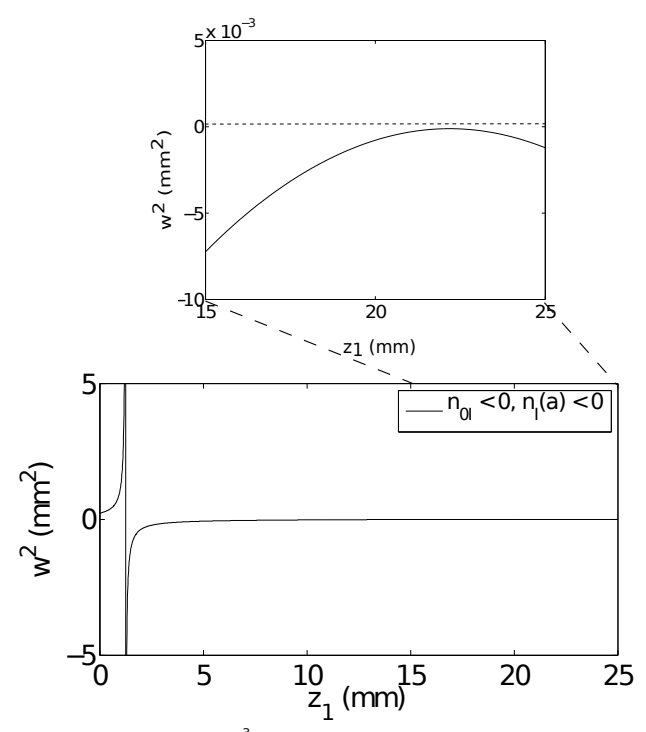

(b)
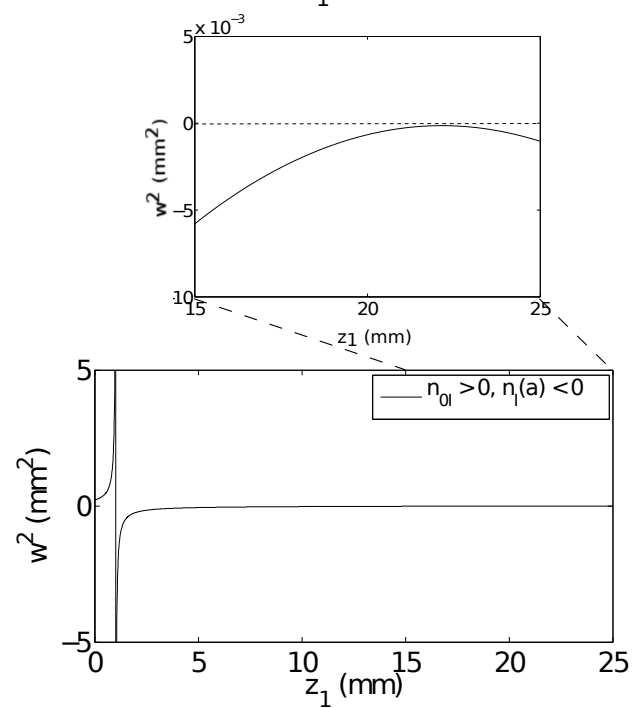

Fig. 2: Evolution of the beam half-width in an active selfoc microlens of thickness $z_{1}=25 \mathrm{~mm}$. Calculations have been made for parameter values of Fig. 1. 


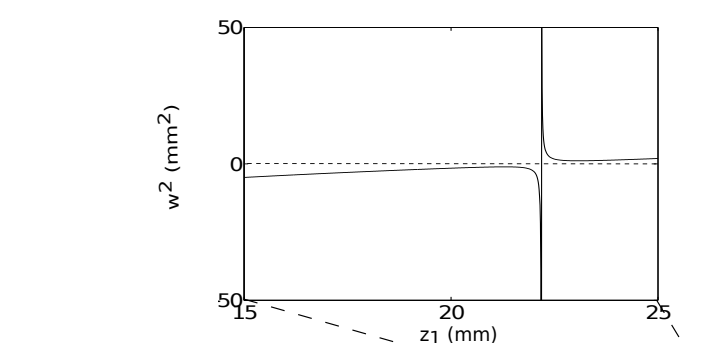

(a)

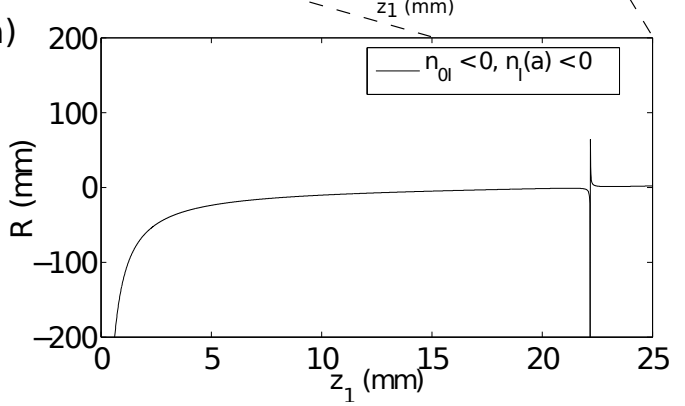

(b)

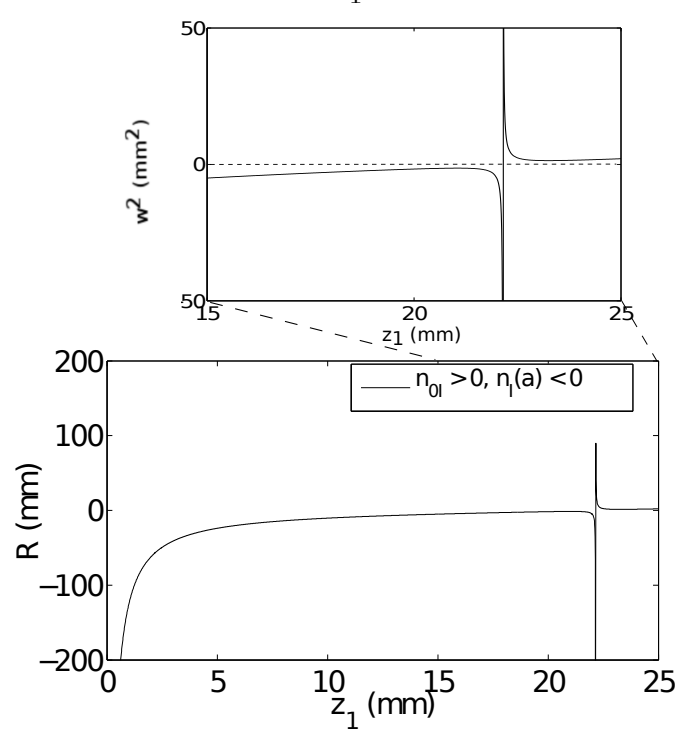

Fig. 3: Evolution of the radius of curvature in an active selfoc microlens of thickness $z_{1}=25 \mathrm{~mm}$. Calculations have been made for parameter values of Fig. 1.

and loss (b). In both cases the radius of curvature presents a similar behaviour: it increases up to the region where the radius of curvature changes its sign, that is, where it converts from convergent to divergent Gaussian beam (focusing).

Figure 4 represents the behaviour of the onaxis relative irradiance of the Gaussian beam propagating along the active selfoc microlens. A strong variation of the relative irradiance value is observed corresponding to the $z_{1}$-plane where the beam changes from convergent to divergent. In Fig. 4(a) we can observe relative irradiance (a)

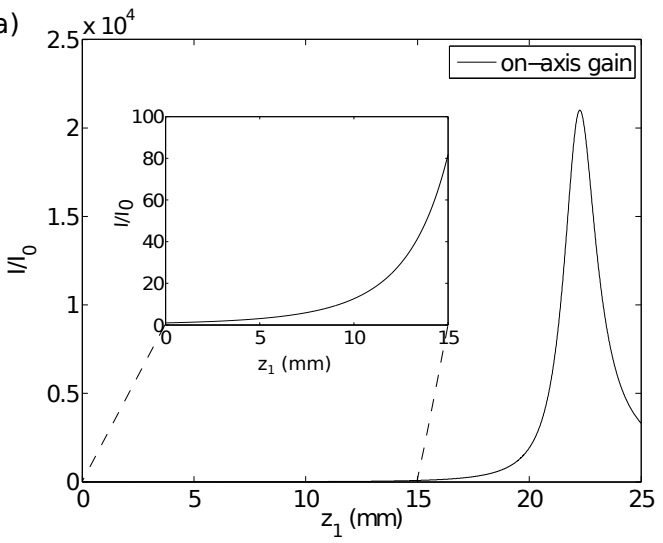

(b)

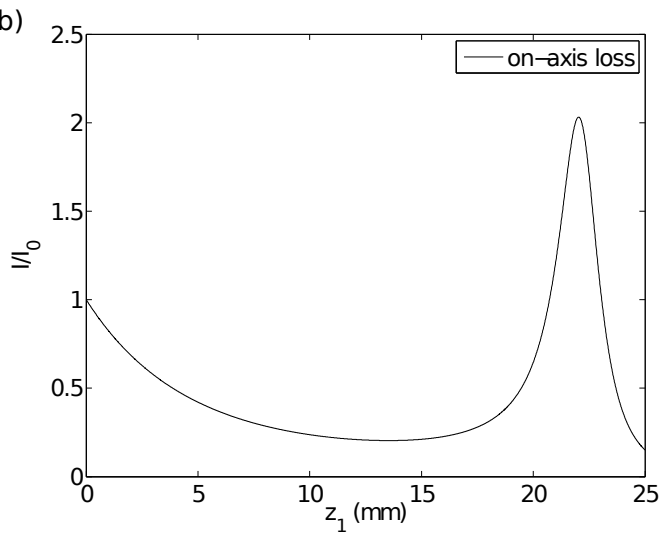

Fig. 4: Evolution of the irradiance of the input Gaussian beam through the active selfoc microlens for (a) on-axis gain and (b) on-axis loss. Calculations have been made for parameter values of Fig. 1.

increases with the axial penetration in the active media due to on-axis gain effects, as shown in enlarged region. When the beam reaches the plane where $\omega^{2}$ changes its sign (see Fig. 2) a flat-top beam is obtained and when the beam achieves the $z_{1}$-plane where it transforms from convergent to divergent a high energy concentration occurs. In Fig. 4(b) the relative irradiance decreases with the axial penetration in the active selfoc microlens passing through beam shaping position up to the $z_{1}$-plane where the beam changes its curvature. At this selfoc length a concentration of irradiance takes place.

\section{Conclusions}

We present here a Gaussian beam transforming system performed by an active selfoc microlens. Light propagation has been analyzed by using a complex refractive index and gradient 
parameter as an analytical extension from expressions for lossless selfoc microlens. Profiles of the real and imaginary part of the refractive index have been analyzed for cases of on-axis gain and loss. The behaviour of the beam halfwidth and the radius of curvature of a Gaussian beam propagating along an active selfoc microlens has been discussed. Finally, relative irradiance for on-axis gain and loss has been evaluated, resulting that when the Gaussian beam reaches the plane where it transforms from convergent to divergent a high energy concentration occurs in the active selfoc microlens.

\section{Acknowledgements}

This work has been sponsored by Xunta de Galicia / Feder (INCITE08PXIB206013PR), Ministerio de Ciencia e Innovación (MAT201018519) and CDTI (SURFALUX SOL-00030930), Spain. Besides, Ana I. Gomez-Varela wants to acknowledge the financial support from the FPU (Formación de Profesorado Universitario) grant 2009 (Ministerio de Educación, Spain). 